\title{
La préhistoire des basses terres de l'Est de l'Uruguay et du Sud du Brésil
}

The Prehistory of the eastern Uruguay and southern Brazil lowlands La prehistoria de las tierras bajas del este de Uruguay y sur de Brasil

José M. López Mazz

\section{OpenEdition}

\section{Journals}

Édition électronique

URL : https://journals.openedition.org/jsa/14302

DOI : $10.4000 /$ jsa. 14302

ISSN : $1957-7842$

\section{Éditeur}

Société des américanistes

\section{Édition imprimée}

Date de publication : 31 décembre 2015

ISSN : 0037-9174

\section{Référence électronique}

José M. López Mazz, «La préhistoire des basses terres de l'Est de l'Uruguay et du Sud du Brésil », Journal de la Société des américanistes [En ligne], 101-1 et 2 | 2015, mis en ligne le 19 avril 2016, consulté le 24 février 2023. URL : http://journals.openedition.org/jsa/14302 ; DOI : https://doi.org/ $10.4000 / j s a .14302$ 


\title{
La préhistoire des basses terres de l'est de l'Uruguay et du sud du Brésil
}

\author{
José M. LóPEZ MAZZ*
}

La façade atlantique de l'Amérique du Sud a été explorée et colonisée depuis le $\mathrm{X}^{\mathrm{e}}$ millénaire av. J.-C., par des chasseurs-cueilleurs très mobiles qui faisaient partie d'une grande sphère d'interaction culturelle. Par la suite, ces groupes devinrent moins mobiles s'attachant à des territoires qui sont autant de paysages culturels spécifiques. Leur économie était alors centrée sur l'exploitation des basses terres, avec la gestion de plantes et de ressources marines. À partir du III millénaire, ces sociétés se complexifièrent, se dotant de systèmes politiques plus forts tandis que les conflits augmentaient. À cette époque, des groupes d'Amazonie (arawak et guarani) arrivèrent dans la région. Les tribus Charrúas et Guenoa/Minúan des XVI et $\mathrm{XVII}^{\mathrm{e}}$ siècles semblent être les descendants historiques de ce long processus. [Mots-clés : basses terres, chasseurs-cueilleurs, sociétés complexes, monticules, préhistoire de l'Uruguay.]

La prehistoria de las tierras bajas del este de Uruguay y sur de Brasil. La fachada atlántica de Sudamérica fue objeto de exploración y colonización humana desde el decimo milenio antes de Cristo, por grupos de cazadores-recolectores de gran movilidad que formaban parte de una extensa esfera de interacción social y cultural. Con el tiempo estos grupos pierden movilidad y desarrollan una territorialidad caracterizada por paisajes culturales singulares y por la explotación de los recursos de las tierras bajas; con énfasis en la gestión de plantas y en los recursos marinos. Desde el tercer milenio antes de Cristo, estos grupos se vuelven más complejos, con sistemas políticos más fuertes e importantes niveles de conflicto. En esta época llegan a la región grupos amazónicos (arawak y guaraníes). Las tribus charruas y güenoas/minuán de los siglos XVI y XVII parecen ser los descendientes históricos de este largo proceso. [Palabras clave: tierras bajas, cazadores-recolectores, sociedades complejas, montículos, prehistoria de Uruguay.]

The Prehistory of the eastern Uruguay and southern Brazil lowlands. The Atlantic slope of South America was explored and colonised from tenth Millennium before J.C. by hunter gatherers groups. These groups with high mobility are part

* Departamento arqueología, facultad de Humanidades/CURE/UdelaR, SNI/ANII Universidad de la República [lopezmazz@yahoo.com.ar]. 
of an extended sphere of cultural interaction. Later they became less mobile and produced a specific cultural landscape. Their economy was based on products, managed plants and marine resources of the lowlands. From the third millennium before the era these inhabitants acquired some degree of complexity with a strong political system able to withstand social conflict. At the beginning of this period people arrived from Amazonia (Arawak and Guarani). Charrua and Güenoa/Minuan tribes (well known in the sixteenth and seventeenth centuries) were the historical heirs of this long process. [Key words: lowlands, hunter-gatherers, complex societies, mounds, prehistory of Uruguay.]

Dès le $\mathrm{X}^{\mathrm{e}}$ millénaire av. J.-C., le versant atlantique méridional de l'Amérique du Sud a été le théâtre d'une colonisation humaine (Meneghin 2006 ; Suárez et López Mazz 2003 ; Suárez 2010 ; López Mazz 2013). Au cours des millénaires suivants, des développements sociaux, culturels et technologiques préhistoriques tout à fait singuliers y ont eu lieu. Néanmoins, dans la littérature classique, cette région est peu connue et, dans le Handbook of South American Indians, elle apparaît sous l'appellation d' " aire marginale », pour bien la distinguer d'autres auxquelles étaient attribuées une identité archéologique et un développement culturel plus affirmés (Steward 1946).

Pourtant déjà à la fin du XIX ${ }^{\mathrm{e}}$ siècle, Francisco Bauzá (1885-1897) et José H. Figueiras (1892) signalaient l'existence de traces d'un peuplement préhistorique dans les basses terres méridionales d'Amérique du Sud, caractérisé par des activités de chasse et de collecte et, plus tard, par la construction de monticules en terre. Ces tertres furent alors dénommés « cerritos de indios » appellation qu'ils ont conservée jusqu'à nos jours (Figure 1). D'autres structures préhistoriques similaires furent identifiées dans le delta du Parana, le long du fleuve Uruguay (Lothrop 1932) et le long du Río de la Plata (Serrano 1972).

Dans les années 1960 et 1970, commencèrent les premières recherches systématiques. Elles furent le fait de chercheurs brésiliens (Naue, Schmitz et al. 1968 ; Copé Moehlecke 1991 ; Schmitz 1976) et uruguayens (Prieto et al. 1970 ; Schmitz et Baeza 1980). Ceux-ci interprétèrent ces monticules comme étant des structures résidentielles domestiques permettant aux chasseurs-cueilleurs d'habiter dans des environnements inondables. Ces groupes furent alors assimilés à la tradition culturelle des chasseurs " pampeanos » (Taddei 1987), caractérisée, en Argentine, en Uruguay et dans le Sud du Brésil, par une technologie lithique comprenant des pointes de flèches, des boleadoras (boules en pierre) et, à une période plus récente, par l'usage de chevaux (Schmitz 1976).

En 1987, un projet public de sauvetage archéologique, rendu nécessaire par la croissance de la culture du riz dans une partie de la région, marque le départ d'une nouvelle étape de la recherche ${ }^{1}$. Les travaux qui suivirent ont inclus plusieurs

1. La « Comisión de Rescate Arqueológico de la Cuenca de la Laguna Merín » (CRALM) était un projet de sauvetage archéologique chargé de dresser un état des lieux sur à peu 


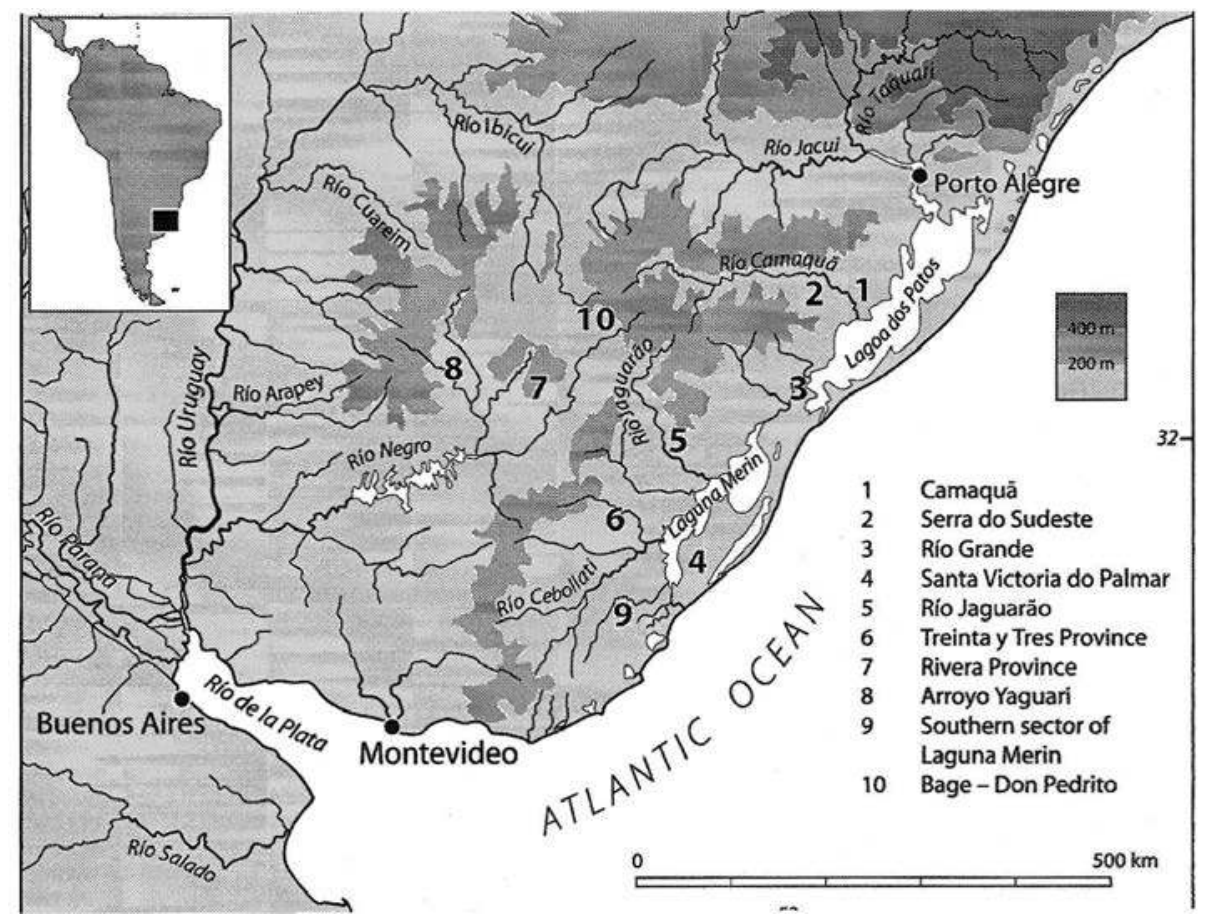

Fig. 1 - Carte de la région ici étudiée localisant les « cerritos de indios ».

prospections régionales et des fouilles stratigraphiques. Commencèrent à développer dans la foulée des tentatives de reconstitution des paléo-environnements des basses terres, ce pour mieux comprendre les comportements humains du passé. Ces travaux étaient fondés sur les concepts et les principes méthodologiques mis au point ailleurs pour l'étude des rapports entre changements environnementaux et transformations culturelles (López Mazz et Bracco 1994 ; López Mazz 2001 ; Bracco 2006).

Dans ce contexte, la vision des chasseurs-cueilleurs considérés comme de stricts primitifs changea peu à peu, et une nouvelle image se dessina, où les sociétés apparurent plus complexes qu'on ne le pensait, avec une organisation sociale, un sens de la territorialité, une économie et un système politique plus développés que prévu (López Mazz et Bracco 1994 ; López Mazz 2001).

D'un autre côté, on commença à s'interroger sur l'identité des Indiens qui habitaient la région au moment des conquêtes espagnole et portugaise. Quel

près $5700 \mathrm{~km}^{2}$ de basses terres. Il s'agissait d'une action coordonnée entre le Ministerio de Educación y Cultura et l’Universidad de la República (1987-2002). 
rapport avaient-ils avec les populations préhistoriques des basses terres du Cône Sud, et du reste de l'Amérique du Sud?

Au cours des 25 dernières années, les recherches archéologiques dans le secteur ont été menées en lien avec les communautés universitaires et ont intégré de nouvelles techniques analytiques (datations ${ }^{14} \mathrm{C}$, zooarchéologie, études sédimentaires, analyses des technologies lithiques et céramiques, travaux paléobotaniques, d'anthropologie biologique, etc.). Parallèlement, s'est développé un nouvel intérêt pour les études ethnohistoriques, orientées vers l'identification des populations indiennes tardives, descendant, ou non, des sociétés préhistoriques (Bracco 1998).

Le principal objectif de ces pages est d'offrir une vue d'ensemble de la préhistoire de cette région, jusqu'à aujourd'hui fort peu connue hormis de quelques spécialistes.

\section{Un environnement très dynamique qui conditionne les conduites humaines}

Le paysage de la région est dominé par des basses terres très étendues et des lagunes proches de l'océan, au milieu desquelles des collines et des terrasses ont été occupées et réoccupées pendant des millénaires. Cet environnement est limité, d'un côté, par la forêt de palmiers Butia odorata et, de l'autre, par la côte atlantique. La lagune la plus grande est la Laguna Merín, entourée de près de $5700 \mathrm{~km}^{2}$ de basses terres, qui s'inondent partiellement ou complètement pendant une grande partie de l'année. Bien qu'ayant souffert de dramatiques changements au cours de l'Holocène, la côte atlantique est riche en ressources marines, comprenant en particulier de grands mammifères (Otaria flavensces et Arctocephalus australis) qui forment des colonies au Cabo Polonio entre le mois de septembre et la fin du mois de mars. Les palmiers, formant de petites forêts, mûrissent en mars et produisent une noix qui était utilisée pour fabriquer de la farine par les Indiens au XVIII ${ }^{\mathrm{e}}$ siècle (César 1981).

Il y a 12000 ans, le paysage était cependant fort différent, notamment parce que le Río de la Plata n'existait pas. À sa place, il avait le paléo-Paraná, un fleuve beaucoup plus étroit qui, en débouchant dans l'océan Atlantique à la hauteur de la plateforme marine, formait un delta (Ayup-Zouain 2006 ; Bracco et al. 2011 ; Figure 2). Cette zone deltaïque devait faciliter la circulation des hommes entre le nord et le sud de l'actuel Río de la Plata (Miotti 2006). Il est important de noter que le bras le plus septentrional et le plus profond (autour de -30/-20 m snm actuel) de ce delta suivait un dessin proche de l'actuelle côte uruguayenne (Ayup-Zouain 2006 ; Bracco et al. 2011).

La fin du Pléistocène et le début de l'Holocène furent marqués par un changement des conditions climatiques, notamment l'augmentation de la température de près de $3^{\circ} \mathrm{C}$ et le développement des plaines alluviales, des lagunes et des milieux aquatiques (Cavalloto, Violante et Parker 2004 ; García Rodríguez et al. 2011). 


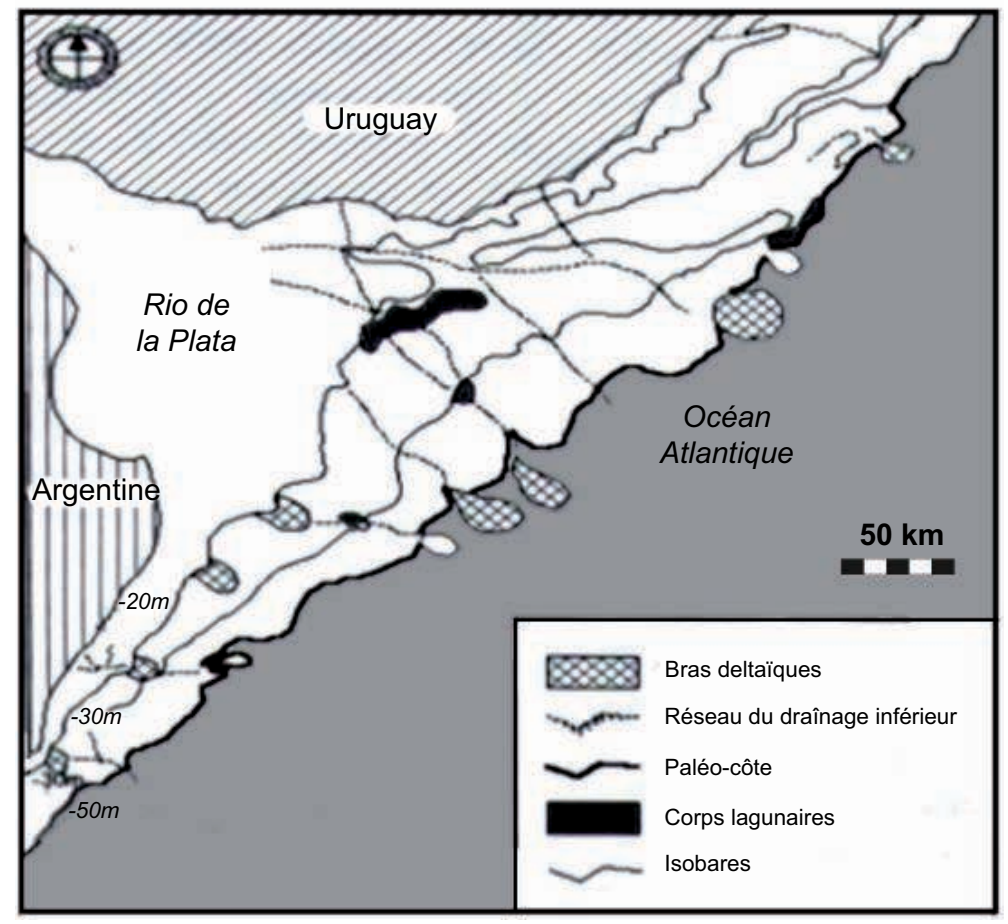

Fig. 2 - Paléographie du bassin du Río de la Plata, vers 9000-4000 av. J.-C. (Ayup Zouain 2006).

Le paysage du post-Pléistocène était ainsi composé d'une mosaïque de zones écologiques, conditionnées par la latitude, la topographie et l'influence marine, avec un développement de basses terres (Cavalloto, Violante et Parker 2004 ; Iriarte 2006) et d'une forêt saisonnière près des fleuves et des cours d'eau (Leal et Lorscheitter 2007).

À partir de 15500 av. J.-C., le niveau de la mer monta depuis -120 m snm actuel jusqu'à submerger les plaines maritimes vers 4000 av. J.-C. (Bracco et al. 2011). Cette transgression marine provoqua la réduction des terres disponibles pour l'habitat et noya l'ancienne ligne de côte sur laquelle étaient implantés les premiers sites archéologiques. Les études paléobotaniques à l'est de l'Uruguay suggèrent que le Pléistocène final (entre 12810 et 8000 av. J.-C. environ) se caractérisait par des conditions froides et arides, ainsi que par des prairies riches de plantes en C3 (Iriarte 2006). Avec l'apparition de conditions plus chaudes et plus humides au début de l'Holocène (vers 7450-4620 av. J.-C.), les basses terres se développèrent, comme l'indiquent la formation de tourbières, la croissance d'espèces adaptées à des conditions humides et le remplacement, dans les prairies, des Pooideae (C3) par des Panicoideae (C4) (ibid., p. 27-28). 
La structure écologique et le paysage du début de l'Holocène ont orienté l'organisation économique et la mobilité des chasseurs-cueilleurs. La montée des eaux, la réduction des terres aptes à l'occupation humaine, aussi bien que la circonscription des ressources critiques, au milieu des étendues de basses terres, ont été la clé d'une nouvelle forme d'occupation de l'espace. Les études paléobotaniques suggèrent que ces conditions ont pu déclencher l'occupation intensive des basses terres (Bracco et al. 2005).

Les changements du régime d'inondation (entre eau douce et eau salée) ont été décisifs aussi pour l'Holocène moyen et, encore une fois, responsables de nouvelles formes d'occupation de l'espace des sociétés préhistoriques. Ces transformations se sont produites en même temps qu'un refroidissement et qu'une aridification relative des conditions climatiques, accompagnés d'une baisse du niveau de la mer et de la formation de champs de dunes côtières. Les datations au ${ }^{14} \mathrm{C}$ du niveau sous les dunes du site archéologique de Cabo Polonio permettent de situer ce changement climatique vers 5000 av. J.-C. (López Mazz et al. 2009). Aux alentours de 1000-500 av. J.-C., les conditions climatiques s'améliorèrent, devenant plus chaudes et plus humides, pour se stabiliser et devenir similaires à celles d'aujourd'hui (Bracco et al. 2005).

L'émergence de la culture préhistorique connue comme celle des «constructores de cerritos » (les bâtisseurs de monticules) (López Mazz et Bracco 1994) est très liée à ce changement de la structure écologique et aux variations de la disponibilité des ressources naturelles des environnements inondables. Les communautés - très variables - de plantes et de faune ont fourni l'essentiel des aliments des sociétés. Durant 10000 ans, les basses terres ont été le lieu d'expériences singulières entre les humains, l'eau, les plantes et les animaux, tout en donnant naissance à une économie originale et à des rapports sociaux particuliers.

Les recherches archéologiques sur les premiers peuplements du versant atlantique de l'Amérique du Sud se heurtent à des problèmes engendrés par les conditions du milieu. D'une part, la montée du niveau de la mer au cours de l'Holocène a noyé les plus anciens sites archéologiques. D'autre part, et plus récemment, entre circa 4000 et 3000 av. J.-C., la formation de dunes côtières a également enseveli des sites sous d'épaisses couches de sable. Dans ces contextes, les fouilles archéologiques doivent adopter des techniques compliquées ou se dérouler en profitant de situations exceptionnelles et au gré de leur apparition.

Le site archéologique d'Urupez sur le Cerro de los Burros (département de Maldonado) est localisé sur une colline et associé à un affleurement de rhyolithe qui a fourni la roche pour confectionner des outils. Depuis quelques années, des pointes de type « queue de poisson » $(\mathrm{PQP})$ ont été collectées en surface, tandis qu'une pointe de quartz blanc a été trouvée dans une fouille stratigraphique, avec d'autres outils lithiques ovales et des éclats de rhyolithe, de quartz, de jaspe et de silex. Des charbons culturels associés ont fourni des datations ${ }^{14} \mathrm{C}$ 
entre 10000 et 9000 av. J.-C. (Meneghin 2006). Ce site étant localisé près de la côte, non loin de l'océan Atlantique, il est possible que vers 11000-9000 ans av. J.-C., à l'époque du paléo-delta, il ait été proche du bras nord de ce dernier.

Le site archéologique de Cabo Polonio est localisé face à l'océan, près de l'ancien bras nord du paléo-delta. Il s'agit d'un cap et de quelques îles abritant des colonies de mammifères marins (Otaria flavensens et Arctocephalus australis). Des fouilles ont été réalisées là dès 1991 : on y a trouvé différentes couches de sable d'origine éolienne (López Mazz et al. 2009). Celles de couleur sombre concentrent le matériel culturel, constituant ainsi les niveaux d'occupation humaine (Figure 3). D'autres couches, plus claires, signalent les niveaux d'abandon du site. Ces fouilles ont permis de récupérer des restes de poisson et de coquilles, mais aussi de la faune terrestre, tels des cervidés et des rongeurs, ainsi que de l'œuf de nandou (Rhea americana), la présence de ce dernier indiquant que l'occupation qui lui correspond a eu lieu au début du printemps. Bien que des pointes PQP aient été trouvées en surface de ce site, seuls quelques éclats de quartz et de granite ont été récupérés dans le niveau le plus ancien (ibid.).

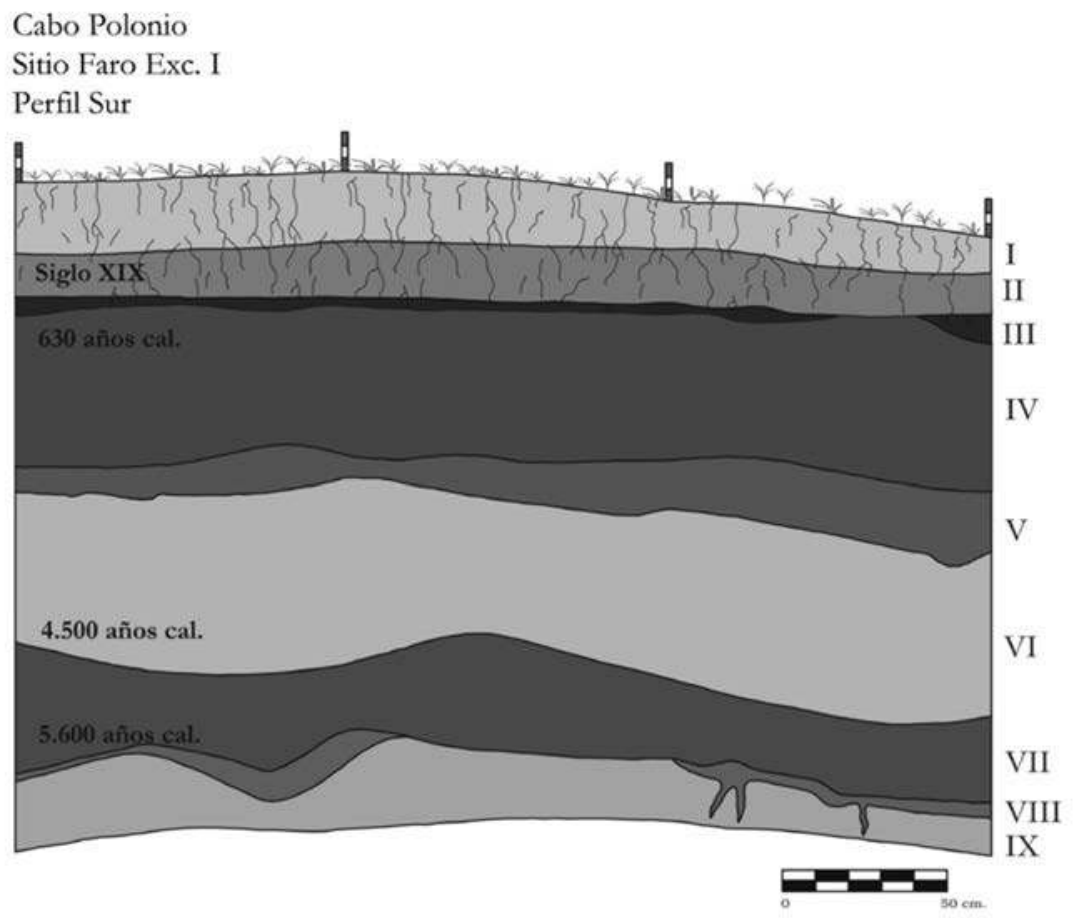

Fig. 3 - Stratigraphie du site Cabo Polonio. 
Plusieurs niveaux archéologiques superposés sont datés entre 70006000 av. J.-C. et le XIV siècle, époque de l'arrivée des Espagnols et des Portugais dans la région. Toutefois, si aucun matériel susceptible d'être daté n'a encore été trouvé dans le niveau le plus ancien, trois échantillons de charbons culturels de la couche au-dessous des dunes ont été datés entre 2400 et 2900 av. J.-C. (López Mazz et al. 2009). On peut signaler que le niveau le plus ancien est similaire à d'autres couches archéologiques du Nord du pays datées d'environ 8500 av. J.-C. (Suárez 2010). L'eau affleure actuellement sous cette couche, ce qui suggère que le moment d'occupation du site correspondait à un niveau de la mer plus bas qu'aujourd'hui.

Le début de l'Holocène se caractérisa par le réchauffement progressif du climat et par plus d'humidité générale. Peu à peu, l'ancien delta du paléo-Paraná disparut sous les eaux, laissant place au Río de la Plata en cours de formation. Alors que beaucoup d'implantations humaines furent submergées, celles situées sur des terrasses continuèrent à être occupées de façon presque continue.

Le site de Ríncón de los Indios est stratégiquement localisé sur les collines de Potrero Grande, au nord de la Laguna Negra, ce qui permettait le transit au nord vers les terres basses d'India Muerta, un des plus anciens centres préhistoriques régionaux. Le site se trouve à $12 \mathrm{~km}$ de l'océan Atlantique, d'où venaient quelques ressources animales et minérales. Entre environ 6800 et 5100 av. J.-C., le sommet fut occupé par des chasseurs-cueilleurs dont un segment du cycle annuel de mobilité (sociale et économique) se situait sur la côte (López Mazz 2013). Les études sur l'organisation technologique des populations montrent que celles-ci se fournissaient en matières premières lithiques de qualité - agate, calcédoine et silex - dans le Nord du pays (López Mazz, Gasacue et Piñeiro 2011).

Deux éléments retiennent l'attention pour cette période, tant dans le territoire uruguayen qu'au sud du Brésil. Le premier est l'évolution et la spécialisation des groupes de chasseurs, dont la technologie, caractérisée par la diversification des pointes de projectile en pierre taillée, peut être associée à la disparition des méga-mammifères du Pléistocène. Le deuxième élément est un schéma de mobilité très dynamique avec des campements saisonniers standardisés, dispersés sur une région étendue, qui va du fleuve Uruguay à l'État de São Paulo au Brésil (Suárez 2010 ; Chmyz et al. 2008 ; Días 2012 ; López Mazz 2013). L'homogénéisation et la standardisation des campements - leur disposition spatiale et celle des rejets - ainsi que de la culture matérielle suggèrent l'existence d'un réseau d'échanges et celle d'une sphère d'interaction sociale et culturelle étendue (Días 2012).

Du point de vue de la technologie de chasse, la diversification des formes de pointes de projectile avec plusieurs styles contemporains impose que l'on s'interroge sur les raisons qui ont produit cette variabilité archéologique. En premier lieu, on pourrait attribuer ces variations à l'histoire elle-même et aux 
recyclages successifs qu'ils ont pu subir (Suárez 2010 ; Castiñeira et al. 2011). Cependant, d'autres raisons ont pu fortement influencer ce processus ; elles sont liées aux changements dans la végétation, aux besoins de la chasse dans les basses terres, à la disparition de la grande faune du Pléistocène et à la disponibilité des matières premières lithiques. Il y a enfin des raisons culturelles (symboliques et sociales) qui peuvent en partie avoir provoqué cette variabilité des types (López Mazz 2013).

Les chasseurs de la transition Pléistocène/Holocène utilisaient encore des pointes de type $\mathrm{PQP}$ (Meneghin 2006) mais, en même temps, commençaient à expérimenter de nouveaux types (Hilbert 1991 ; Austral 1995 ; Suárez 2010 ; Días 2012 ; Mentz Ribeiro 1991). Au centre de la question, se trouve le débat sur les systèmes de propulsion qui sont associés aux différentes pointes de projectile. La datation entre 8000 et 7000 av. J.-C. de pointes de projectile plus petites que le type $\mathrm{PQP}$ suggère qu'il y a eu un centre d'innovation technique indépendant, plus ancien dans ces basses terres que dans les autres régions du continent sud-américain (Figure 4).
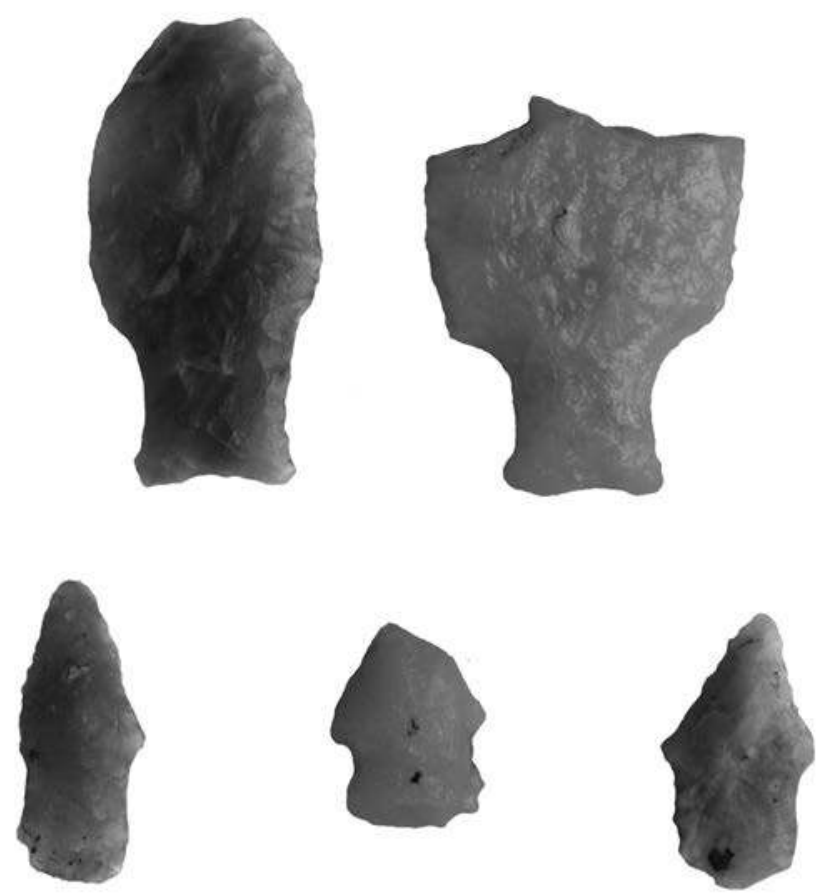

$5 \mathrm{~cm}$

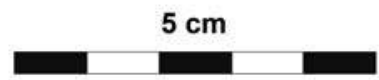

Fig. 4 - Pointes de projectile de la période comprise entre 9000 et 5000 av. J.-C. 


\section{Les chasseurs cueilleurs adoptent l'horticulture (vers 2000 av. J.-C.)}

La période formative de la région est caractérisée par une gestion intensive des plantes, l'élargissement du spectre des espèces animales chassées (et pêchées) et des changements sociopolitiques exprimés dans les sépultures et dans un nouveau paysage culturel. Le modèle socio-économique traditionnellement relié à ce type de société préhistorique est celui de groupes de chasseurs-cueilleurs spécialisés exploitant les riches et très diverses ressources des basses terres. Ce modèle, centré sur la haute productivité (et prévisibilité) des environnements des terres inondables, n'excluait pas l'horticulture (López Mazz et Bracco 1994 ; Figure 5).

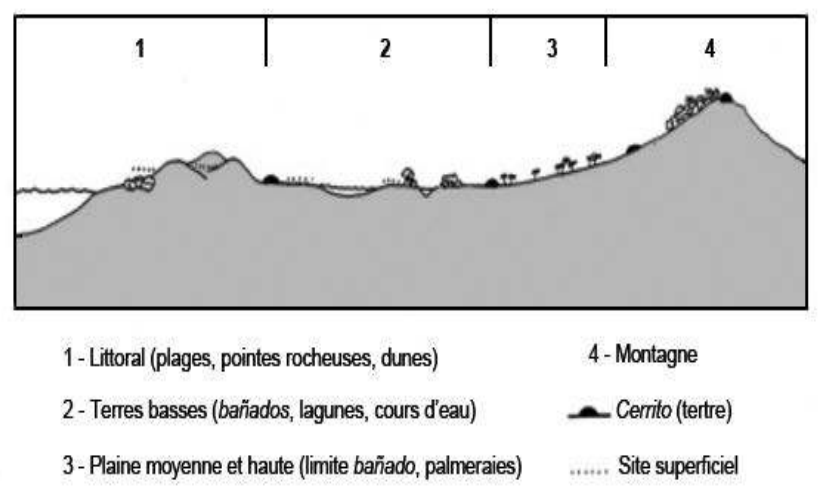

Fig. 5 - Niches écologiques de l'Est de l’Uruguay.

Les recherches paléobotaniques réalisées depuis quelques années ont montré que, parmi les plantes exploitées, se trouvaient le fruit d'un palmier (Butia capitata) et un tubercule (Canna glaucia) (Iriarte et al. 2001, 2004 ; Del Puerto et Campos 1999). À l'époque, apparaît une utilisation particulière du palmier cité, et un outil connu par l'ethnographie comme « cassenoix », qui montre une intensification dans l'exploitation de la noix du fruit de celui-ci (Dabezies et Gazzán 2008). Parmi les plantes domestiquées, ont été identifiés des silicophytolites de Paseolus sp., de Cucurbita et de Zea maiz (Iriarte et al. 2001 ; Del Puerto et Campos 1999), ainsi que des grains d'amidon d'Hypomoea batata et d'Arachis hypogaea (Dabezies 2009 ; López Mazz, Dabezies et Capdepont 2013). La présence de grains d'amidon de l'hallucinogène Datura ferox dans le site archéologique de Laguna de Castillos, pour sa part, laisse imaginer une importante activité rituelle à cette période (ibid.). Cette plante hallucinogène pousse encore aujourd'hui sur les monticules préhistoriques de Laguna Negra et d'India Muerta.

En ce qui concerne les activités de chasse, on constate la haute variabilité des types de pointes de flèches (orientées vers une faune plutôt petite) et l'intensification de l'exploitation des ressources aquatiques (poissons, crustacés, bivalves, 
etc.) (Días 2012 ; López Mazz 2013). Au-delà des témoignages strictement archéozoologiques, on reconnaît des innovations technologiques comme les poids de ligne et/ou de filet de pêche (López Mazz et Gascue 2007).

À l'époque, les sites répartis dans le territoire s'organisent en une véritable hiérarchie et témoignent d'un paysage économique, social et politique plus complexe (López Mazz 1999 ; López Mazz et Gianotti 2001). L’habitat s'oriente vers une semi-sédentarisation et montre une consolidation des cimetières familiaux (Bertoni et al. 2004). L'étude des processus de formation des sites indique clairement la réoccupation régulière des localités les mieux placées dans le paysage, et une intensification de l'activité rituelle exprimée dans différents types d'enterrements (Femenías et al. 1990 ; López Mazz 2001). Parallèlement, le traitement des morts implique davantage de soins apportés aux défunts (Figure 6).

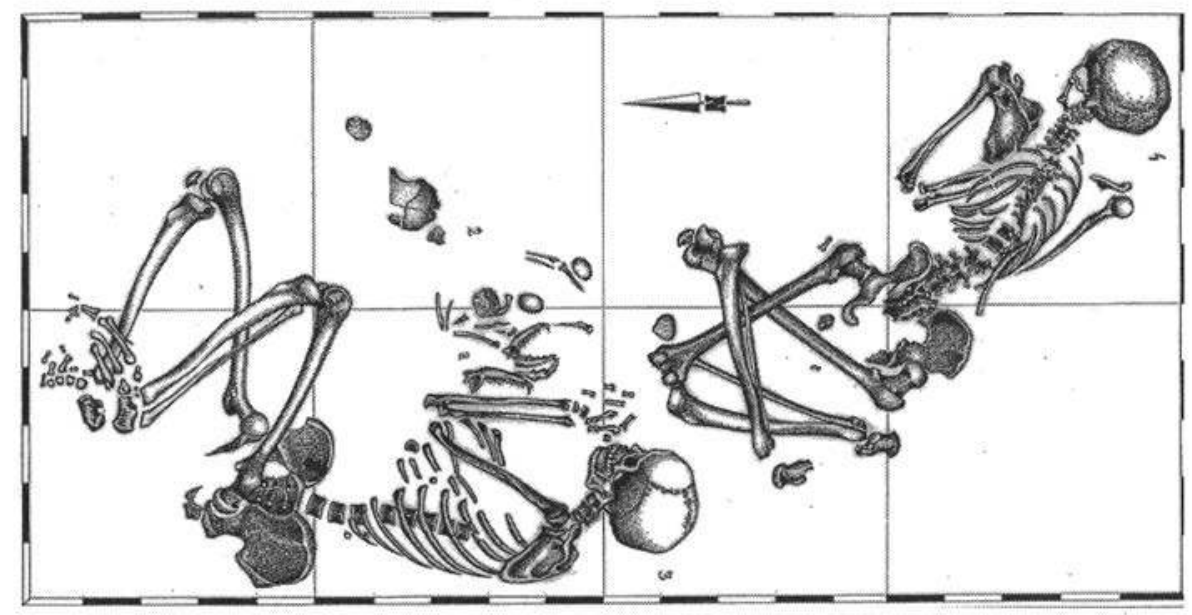

Fig. 6 - Enterrement complexe $(\mathrm{n}=3)$ site Isla Larga (Cabrera 1999).

C'est alors que se produisit une dispersion vers le sud de groupes provenant de l'Amazonie, que l'on peut reconnaître grâce à des céramiques incisées et peintes. Vers 1500 av. J.-C., des peuples liés à la tradition arawak (Politis et Bonomo 2012) et, plus tard, vers le début de notre ère, des groupes guaranis, sont arrivés par les grands fleuves Paraná et Uruguay, les basses terres du Matto Grosso et la côte atlantique (Brochado 1978).

La complexité sociale et politique qui se développe alors dans les basses terres est donc portée par deux éléments complémentaires. D'un côté, elle est stimulée par des logiques internes propres aux processus historiques locaux. De l'autre, l'évolution sociale a aussi été soutenue par des facteurs externes comme l'arrivée des peuples amazoniens. Dans ce contexte, il est plus facile de comprendre le rôle joué par les grands chefs (dont les tombes sont plus grandes 
et plus complexes) et surtout par les conflits et la violence. L'institutionnalisation du conflit apparaît dans les sites de cette période sous la forme de palissades (Iriarte 2006). Des traces de scalpation, d'anthropophagie et de têtes trophées ont été identifiées dans les vestiges fouillés au sud du Brésil (Schmitz 1976) et dans l'Est de l'Uruguay (Gianotti et López Mazz 2009 ; López Mazz et Moreno Rudolph 2013) (Figure 7).

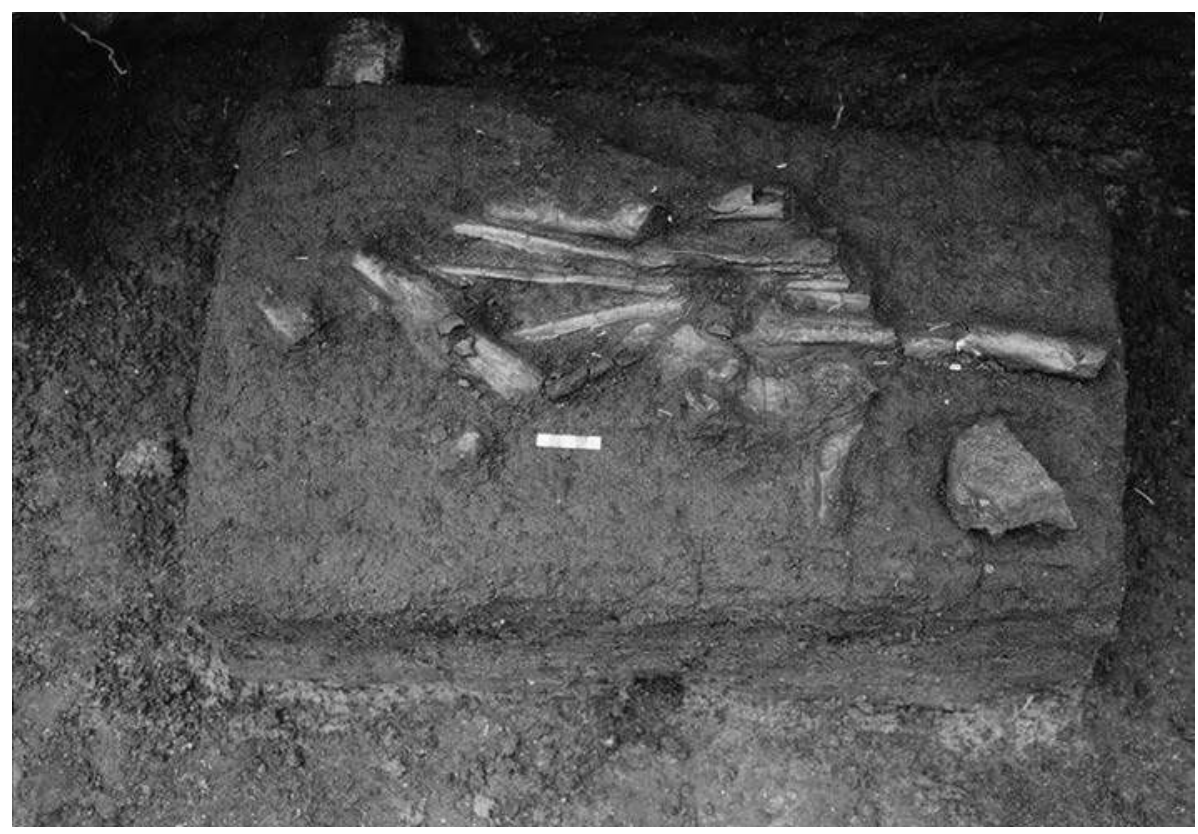

Fig. 7 - Enterrement secondaire avec ocre rouge vers 500 av. J.-C. du site Rincón de Los Indios.

Les grands caciques et les conquêtes espagnole et portugaise (du début de l'ère chrétienne au XVI ${ }^{\mathrm{e}}$ siècle)

À partir du début de notre ère, on assiste à une importante expansion de ces peuples à travers les terres basses et tout le long de la côte atlantique. Dans chaque région, l'occupation de l'espace dut s'adapter à des conditions géographiques locales diversifiées, ce qui produisit des structures particulières d'occupation de l'espace (Bracco 2006 ; Iriarte 2006 ; López Mazz 2001). Parmi les nouvelles zones occupées de façon systématique, il y a le piémont de la Sierra de San Miguel et le bord de la mer, où s'étaient installés auparavant des campements saisonniers et de valeur stratégique, qui permettaient d'exploiter de façon simultanée des ressources provenant de diverses niches écologiques proches (López Mazz et Villarmarzo 2009). 
La violence et les conflits identifiés au niveau archéologique, qui avaient commencé au $\mathrm{I}^{\mathrm{er}}$ millénaire av. J.-C., continuèrent au début de l'ère chrétienne, de façon concomitante avec la pratique de l'anthropophagie et des têtes trophées (Pintos et Bracco 1999 ; López Mazz et Moreno Rudolph 2013 ; Gianotti et López Mazz 2009). La mise en place de cette nouvelle organisation territoriale est marquée par une série de sites hiérarchisés répartis dans différentes zones (López Mazz et Pintos 2000, 2001). Cette organisation suggère l'existence de différents niveaux de décision politique et d'un système social macro-régional avec des populations qui s'alliaient ponctuellement pour résister à des périls externes mais se retrouvaient en conflit à d'autres moments (Dillehay 1996 ; López Mazz 2001).

Une autre manifestation de la complexité des sociétés, soulignée par les chercheurs, est le paysage anthropisé. Celui-ci s'est constitué lentement sur plusieurs millénaires, mais c'est à cette époque qu'il se consolide. Il est à la fois le support et le produit d'une conduite de contrôle du territoire, avec la monumentalité associée aux activités cérémonielles et une stratégie d'intervisibilité entre les localités et les concentrations de ressources (lagunes, forêts de palmiers, etc.) (Figure 8) (Andrade et López Mazz 2000 ; Gianotti et López Mazz 2009 ; López Mazz 2001).

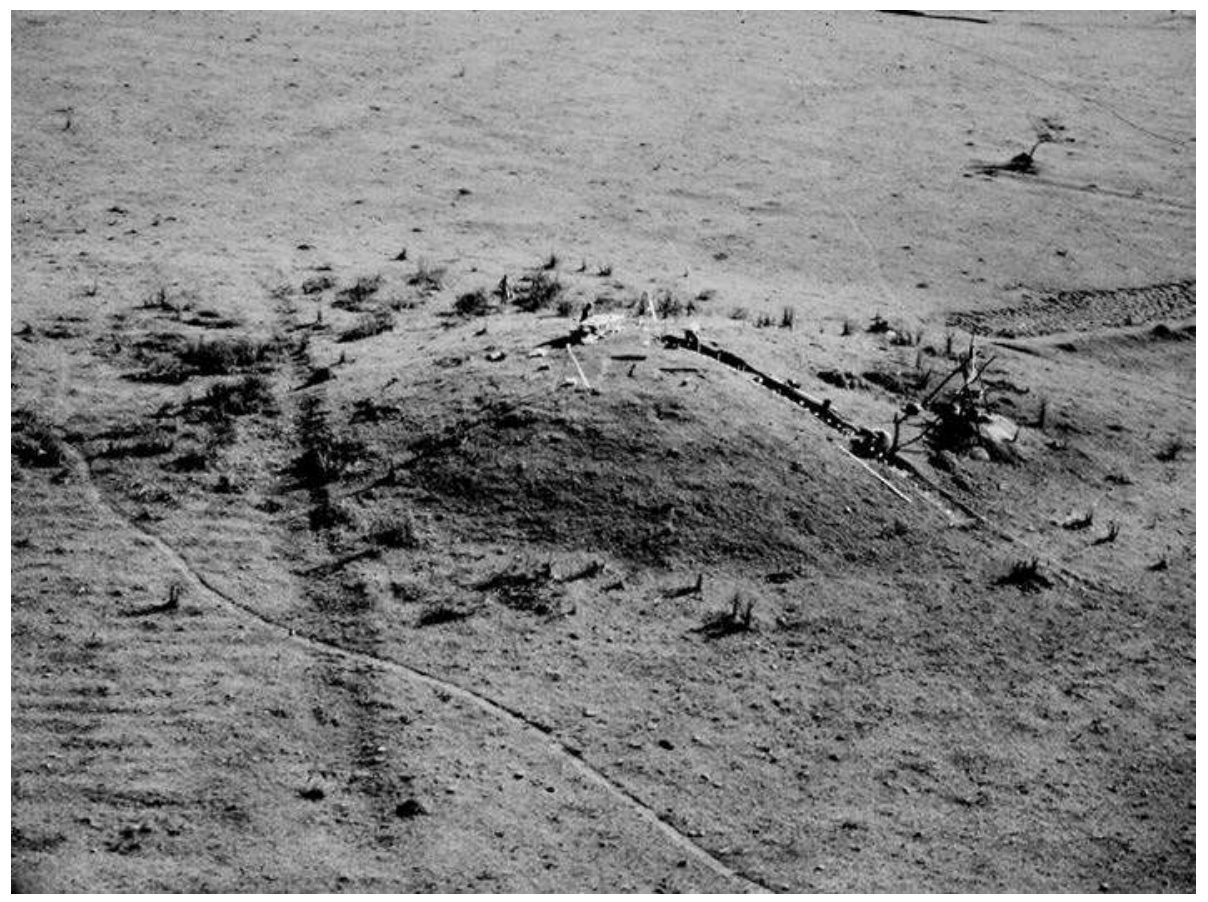

Fig. 8 - « Cerrito de indios », zone d'India Muerta. 
Les chroniqueurs de l'époque coloniale confirment beaucoup d'aspects de la complexité sociale, que l'on peut reconstituer à partir des données archéologiques, notamment ce qui concerne la figure de ces caciques qui vont être en première ligne face aux conquêtes espagnole et portugaise du XVI ${ }^{\mathrm{e}}$ siècle (López Mazz et Bracco 2010). Les Indiens vont très rapidement adopter l'élevage, une transformation peut-être favorisée par les expériences que les anciens chasseurs avaient développées sur des troupeaux de cervidés, lesquels avaient été la principale source de protéines pendant des millénaires. Des chiens, souvent enterrés dans les " cerritos », ont pu aider à une meilleure gestion des cervidés (López Mazz 2001).

L'arrivée des premiers conquérants intensifia les conflits qui existaient entre les Indiens habitant la région depuis des millénaires et les peuples guaranis qui les avaient repoussés vers l'intérieur des terres. Comme dans d'autres zones d'Amérique, l'opposition entre les groupes autochtones profita aux conquérants qui développèrent des liens très étroits avec les Guarani.

Les Charruas et les Güenoas, connus également sous le nom de Minuanes, résistèrent à la fondation des premières villes européennes. Ce sont les descendants historiques des populations préhistoriques. Au XVIII ${ }^{\mathrm{e}}$ siècle, les Güenoas furent expulsés vers le nord et se fixèrent au sud du Brésil. Métissés, ils adaptèrent leurs traditions techniques et culturelles au mode de production européen centré sur l'élevage des bovidés, des chevaux et des ânes (López Mazz et Bracco 2010). Du processus social de métissage émergea la figure du gaucho, personnage emblématique et chevalier renommé des plaines du bassin du Río de la Plata.

En 1833, le nouvel État uruguayen mena une guerre d'extermination contre les derniers Charrúas et Güenoas/Minúan, donnant ainsi naissance au stéréotype d'un Uruguay seul pays de l'Amérique sans Indiens.

\section{Conclusions}

La transition Pléistocene/Holocène a définitivement transformé les milieux et les paysages de la façade atlantique méridionale de l'Amérique du Sud avec : a. des variations du niveau de la mer et des conditions climatiques ; b. le développement des basses terres (et d'un système de lagunes côtières) et c. des changements dans la structure écologique des ressources critiques disponibles pour la survie.

Dans ce contexte, les plus anciennes sociétés de chasseurs-cueilleurs ont dû s'adapter à de nouvelles conditions environnementales. Ce processus d'ajustements et de changements adaptatifs a donné lieu à la première tradition technologique de chasseurs-cueilleurs proprement locale, connue sous le nom de tradition Ombú (vers 8000 av. J.-C.). Dépendantes des matières premières lithiques disponibles et destinées à une faune plus petite (en grande mesure associée aux environnements inondables), les pointes de projectile en pierre qui apparaissent alors variées constituent le produit le plus visible de la réponse humaine à la mise en place de l'Holocène. 
Les paysages anthropiques et culturels bâtis sur la longue durée et qui émergèrent dans les basses terres à partir de $3000 \mathrm{av}$. J.-C. devinrent peu à peu un produit « socio-naturel » qui reflète la disponibilité des espaces à habiter et a permis de développer les activités de l'économie domestique. L'occupation répétée des terrasses (sédimentaires et structurelles) donna naissance à un paysage social à fort caractère territorial (composé de milliers de cerritos de indios) comportant des aspects économiques fondamentaux, le tout au service de systèmes politiques plus ou moins centralisés (Kolb 1994).

À partir de 1500 av. J.-C., la gestion intensive de l'environnement intégra différentes plantes, parmi lesquelles des espèces sauvages (palmiers, tubercules) et d'autres cultivées (maïs, Phaseolus, calebasses, patate douce, cacahuète). L'organisation économique détermina un calendrier saisonnier, de façon à exploiter un écotone riche et très varié, tout en faisant une large place aux ressources côtières (poissons, mammifères marins, coquilles, oiseaux, etc.).

L'évolution des sites vers une occupation plus prolongée donna lieu, à partir de 500 av. J.-C., à l'émergence de localités de premier ordre, où l'activité rituelle (politique et religieuse) était fortement associée à la mort. Les structures funéraires se combinèrent également avec de grands terrassements de dimensions vraiment monumentales.

Le contrôle des ressources naturelles a évolué sous l'influence des changements de l'environnement: depuis des formes de territorialité dynamiques propres aux chasseurs vers d'autres formes moins mobiles et centrées sur les lagunes, les palmeraies et, finalement, les pâturages aptes à l'élevage.

Les tribus Charrúas et Güenoas/Minúan qui, aux $\mathrm{XVI}^{\mathrm{e}}$, $\mathrm{XVII}^{\mathrm{e}}$ et $\mathrm{XVIII}{ }^{\mathrm{e}}$ siècles, construisaient toujours des «cerritos » résistèrent avec leurs flèches en pierre taillée à la conquête européenne et continuèrent sûrement à pratiquer l'anthropophagie et la scalpation. Ils étaient en réalité, au moins culturellement, les descendants historiques des diverses sociétés de la préhistoire locale. *

* Manuscrit reçu en octobre 2013, accepté pour publication en février 2015.

Je remercie Stéphen Rostain pour avoir corrigé ce texte ainsi que les illustrations qui l'accompagnent, et pour avoir lancé une coopération entre archéologues français et uruguayens. Je suis aussi reconnaissant aux collègues de la Maison de l'archéologie et de l'ethnologie René-Ginouvès pour m'avoir amicalement reçu dans leur laboratoire ( « Archéologie des Amériques » UMR 8096). C'est à la générosité du programme ECOS-Sud que l'on doit l'établissement d'une collaboration archéologique France-Uruguay. 


\section{Références citées}

ANDRADE Lima Tania et José LóPEZ MAZZ

2000, « El surgimiento de sociedades complejas en la prehistoria del litoral Atlántico meridional de Sudamérica », Boletín de antropología americana, 17-19, p. 129-175.

Austral Antonio

1995, « Los cazadores recolectores del sitio estratificado de Paypaso hace 10 mil años », in Mario Consens, José M. López Mazz et María del Carmen Curbelo (éd.), Arqueología en Uruguay, VIII Congreso Nacional de Arqueología de Uruguay, Banco Comercial, Montevideo, p. 212-217.

Ayup-Zouain Ricardo N.

2006, «Evolución paleográfica y dispersión de sedimentos del Río de la Plata », in Rodrigo Menafra, Lorena Rodríguez-Gagello, Fabrizio Scarabino et Daniel Conde (éd.), Bases para la conservación y el manejo de la costa uruguaya, Vida Silvestre, Montevideo.

BAUZÁ Francisco

1885-1897, Historia de la dominación española en Uruguay, 3 vol., Biblioteca Artigas, Montevideo.

Bertoni Bernardo, Gonzalo Figueiro, Gabriel Cabanas, James Mc Donough

2004, «Primeras secuencias de ADN mitocondrial de indígenas prehistóricos del Uruguay », in Laura Beovide, Isabel Barreto et Carmen Curbelo (éd.), X Congreso uruguayo de arqueología. La arqueología uruguaya ante los desafíos del nuevo siglo, Asociación Uruguaya de Arqueología, Montevideo [CD-ROM].

Bracco Diego

1998, Guenoas, Ministerio de Educación y Cultura, Montevideo.

BRACCO Roberto

2006, « Montículos en la cuenca de la Laguna Merín: tiempo, espacio y sociedad », Latin American antiquity, 17 (4), p. 511-540.

Bracco Roberto, Laura Del Puerto, Hugo Inda et Carola CastiÑeira

2005, « Middle-Late Holocene cultural and environmental dynamics in the east of Uruguay », Quaternary international, 132, p. 37-45.

Bracco Roberto, Felipe Garcia, Hugo Inda, Laura Del Puerto

2011, « Niveles relativos del mar durante el Pleistoceno final-Holoceno en la costa de Uruguay ", in El Holoceno en la costa uruguaya, Comisión Sectorial de Investigación Científica/Universidad de la República, Montevideo, p. 65-92.

Brochado José P.

1978, Ecological model of the spread of Amazonian pottery, thèse de doctorat en anthropologie, University of Stanford, Stanford.

CABRERA Leonel

1999, « Funebria y sociedad entre los constructores de cerritos del este uruguayo », in José M. López Mazz et Mónica Sans (éd.), Arqueología y bioantropología 
de las tierras bajas, Universidad de la República, Faculdad de Humanidades y Ciencias de la Educación, Montevideo, p. 63-80.

Castiñeira Carola, Marcelo Cardillo, Judith Charlin, Jorge Baeza

2011, « Análisis de morfometría geométrica de las puntas Cola de Pescado del Uruguay », Latin American antiquity, 22 (3), p. 335-358.

Cavallotto José Luis, Roberto A. Violante et Gerardo Parker

2004, «Sea-level fluctuation during the last 8600 years in the de la Plata river (Argentina) », Quaternary international, 114, p. 155-165.

CÉSAR Guillermino

1981, Primeros cronistas de Río Grande do Sul 1605-1801, Universidades de Río Grande do Sul, Porto Alegre.

Chmyz Igor, Eliane Maria Sganzerla, Jonas Elias Volcov, Eloi BorA

2008, « A arqueología da área da LT 750 kV Ivaipora-Itaberá III, Paraná-Sao Paulo », Arqueología-Revista do Centro de estudos e pesquisas arqueológicas/UFPR, 5, p. 1-305.

\section{Copé Moehlecke Silvia}

1991, « A ocupacão pré-colonial do sul e do sudeste do Río Grande do Sul », in Arno A. Kern (éd.), Arqueología pré-historia do Rio Grande do Sul, Editora Mercado Alberto (Série Documenta 26), Porto Alegre, p. 191-220.

DABEZIES Juan Martín

2009, « Elaboración de una colección de referencia de almidones con utilidad arqueológica », in Tirso Bourlot, Damián Bozzuto, Carolina Crespo, Ana Carolina Hecht (éd.), Entre pasados y presentes II. Estudios contemporáneos en ciencias antropológicas, Fundación de Historia Natural Félix de Azara, Buenos Aires.

DABEZIES Juan Martín et Nicolas GAZZÁN

2008, «Etnoarqueología en los tiempos que corren », in OrJIA (éd.), Actas de las I Jornadas de jóvenes en investigación arqueológica: dialogando con la cultura material, t. 1, Compañía Española de Reprografía y Servicios, Madrid.

Del Puerto Laura et Sara CAmpos

1999, « Silicofitolitos: un abordaje alternativo de la problemática arqueobotánica del este de Uruguay ", in Carlos Alberto Aschero, M. Alejandra Korstanje et Patricia Vuoto (éd.), En los tres reinos: prácticas de recolección en el Cono Sur de América, Instituto de Arqueología y Museo/Facultad de Ciencias Naturales e Instituto Miguel Lillo/Universidad Nacional de Tucumán, Tucumán, p. 141-150.

DíAs Adriana

2012, « Hunter-gatherers occupation of south Brazil Atlantic forest. Paleoenvironment and archaeology », Quaternary international, 256, p. 12-18.

Dillehay Thomas

1996, Some speculations about the cerritos of Southern Uruguay, 33 p., ms. 
Figueiras José H.

1892, «Los habitantes prehistóricos del este de Uruguay », in Comisión de exposición histórico-americana (éd.), El Uruguay en la exposición Mundial de Madrid, Memoria, Montevideo.

García Rodriguez Felipe (éd.)

2011, El Holoceno en la zona costera de Uruguay, CSIC, Universidad de la República, Montevideo.

Femenías Jorge, José López Mazz, Roberto Bracco, Leonel Cabrera

1990, « Tipos de enterramiento en estructuras monticulares (cerritos) de la Cuenca de la Laguna Merín (R.O.U.) », Revista do CEPA, 17, p. 345-358.

Gianotti Camila y José López Mazz

2009, « Intensificación ceremonial, espacios rituales y antropofagia en el Rincón de los Indios », in José M. López Mazz et Andrés Gascue (éd.), Arqueología prehistórica uruguaya en el siglo xxi, Biblioteca Nacional/Facultad de Humanidades y Ciencias de la Educación, Montevideo, p. 33-55.

HiLbert Klaus

1991, Aspectos de la arqueología de Uruguay, Verlag Philipp von Zabern (Materialien zur allgemeinen und vergleichenden Archäologie, 44), Mainz am Rhein.

IRIARTE José

2006, «Vegetation and climate change since $14,810{ }^{14} \mathrm{C}$ yr BP in southeastern Uruguay and implications for the rise of early Formative societies », Quaternary research, 65 , p. 20-22.

Iriarte José, Irene Holst, José López MAzz et Leonel CABrera

2001, « Subtropical wet adaptation in Uruguay during mid-Holocene: an archaeobotanical perspective », in Barbara A. Purdy (éd.), The environmental and cultural heritage of wetlands, Oxbow Books, Oxford, p. 51-60.

Iriarte José, Irene Holst, Oscar MArozzi, Claudia Listopard

2004, « Evidence for cultivar adoption and emergent complexity during the mid-Holocene in the La Plata basin », Nature, 432, p. 614-617.

KolB Michael J.

1994, « Monumentality and the rice of religious authority in precontact Hawai' $i$ », Current anthropology, 34 (5), p. 521-547.

LEAL Márcia Grala et Maria Luisa LoRsCheITTER

2007, «Plant succession in a forest on the lower northeast slope of Serra Geral, Rio Grande do Sul, and Holocene paleoenvironments, southern Brazil », Acta botanica brasilica, 21 (1), p. 1-10.

LóPez Mazz José M.

1999, « Construcción del paisaje y cambio cultural en las tierras bajas del este de Uruguay », in José López Mazz et Mónica Sans (éd.), Arqueología y bioantropología de las tierras bajas, Universidad de la República, Faculdad de Humanidades y Ciencias de la Educación, Montevideo, p. 35-62. 
2001, « Las estructuras monticulares (cerritos) del litoral atlántico uruguayo », Latin American antiquity, 12 (3), p. 3-35.

2013, « Early human occupation of Uruguay: radiocarbon database and archaeological implications », Quaternary international, 21, p. 11-22.

López Mazz José M. et Diego Bracco

2010, Minuanes. Apuntes y notas para la arqueología y la historia del territorio Güenoa/Minúan, Librería Linardi y Risso, Montevideo.

López Mazz José M. et Roberto Bracco

1994, « Cazadores colectores complejos de la cuenca de la Laguna Merin », in José Luis Lanata et Alberto Borrero (éd.), Arqueología de cazadores-recolectores: limites, casos y aperturas, Programa de Estudios Prehistóricos, Buenos Aires, p. 1-33.

López Mazz José M., Juan M. DABezies et Irina CAPDepont

2014, « La gestión de los recursos vegetales en la prehistoria de las tierras bajas del este de Uruguay. Un abordaje interdisciplinar », Latin American antiquity, 25 (3), p. 256-277.

López Mazz José M. et Andrés Gascue

2007, « El valle del arroyo Balizas: estructuras monticulares y sitios del litoral atlántico uruguayo », Cazadores recolectores del Cono Sur, revista de arqueología, 2, p. $89-103$.

López Mazz José M., Andrés Gasacue et Gustavo Piñeiro

2011, « Flint procurement strategies of the early hunter-gatherer of eastern Uruguay », in Marta Capote, Susana Consuegra, Pedro Díaz-del-Río et Xavier Terradas, Proceeding of the $2^{\text {nd }}$ International conference of UISPP Commission of flint mining in pre- and protohistoric times (Madrid, 14-17 October 2009), British Archaeological Reports (BAR International Series), Oxford, p. 290-302.

López Mazz José M. et Camila Gianotti

2001, « Construcción de espacios ceremoniales públicos entre los pobladores prehistóricos de las tierras bajas de Uruguay: el estudio de la organización espacial en la localidad arqueológica de Los Indios », Revista de arqueología, 11, p. 87-104.

López Mazz José M. et Federica Moreno Rudolph

2014, « El cambio social en la prehistoria de las tierras bajas del este de Uruguay: la visibilidad arqueológica del conflicto », in José M. López Mazz et Mónica Berón (éd.), Indicadores arqueológicos y bioantropológicos de conflicto y violencia en América Latina, Universidad de la República, Montevideo, p. 3-22.

López Mazz José M., Federica Moreno Rudolph, Eugenia Villarmarzo et Andrés Gascue 2009, « Apuntes para una arqueología costera y del Cabo Polonio », in José López Mazz et Andrés Gascue (éd.), Arqueología prehistórica uruguaya en el siglo XXI, Biblioteca Nacional del Uruguay, Montevideo, p. 39-65.

López Mazz José M. et Sebastian PINTos

2000, « Distribución espacial de estructuras monticulares en la cuenca de la Laguna Negra », in Alicia Durán Coirolo y Roberto Bracco Boksar (éd.), Arqueología 
de las Tierras Bajas, Ministerio de Educación y Cultura, Comisión Nacional de Arqueología, Montevideo, p. 49-58.

2001, «El paisaje arqueológico de la Laguna Negra », in Arqueología uruguaya al fin del milenio, IX Congreso Nacional de Arqueología de Uruguay, Montevideo, p. 175-186.

López MAzz José M. et Eugenia Villarmarzo

2009, «Explotación intensiva de recursos marinos: el caso del este de Uruguay », in José M. López Mazz et Andrés Gascue (éd.), Arqueología prehistórica uruguaya en el siglo XXI, Biblioteca Nacional del Uruguay, Montevideo, p. 13-26.

Lothrop Samuel Kirkland

1932, « Indians of the Paraná Delta, Argentina », in Annals of the New York academy of science, 33, p. 77.

Meneghin Ugo

2006, « Un nuevo registro radiocarbónico $\left({ }^{14} \mathrm{C}\right)$ en el yacimiento Urupez II, Maldonado, Uruguay », Orígenes, 5, p. 1-7.

Mentz Ribeiro Pedro

1991, « Arqueología do Vale do Río Pardo, Rio Grande do Sul, Brasil », Revista do CEPA, 18 (21), p. 1-184.

Miotтi Laura

2006, « La fachada atlántica como puerta de ingreso alternativa de la colonización de América del Sur, durante la transición Pleistoceno/Holoceno », in José Concepción Jiménez López (éd.), $2^{\circ}$ Simposio internacional « El hombre temprano en América », Instituto Nacional de Antropología e Historia, Mexico, p. 155-188.

Naue Guillermo, Ignacio Schmitz et Itala Irene Basile Becker

1968, « Sitios arqueológicos no municipio de Rio Grande do Sul », in Publicacoes avulsas do Instituto anchietano de pesquisas, 1, Instituto Anchietano de Pesquisas, Sao Leopoldo, p. 141-154.

PinTos Sebastián et Roberto Bracco

1999, « Modalidades de enterramiento y huellas de origen antrópico en especímenes óseos humanos. Tierras bajas del Este de Uruguay », in José M. López Mazz et Mónica Sans (éd.), Arqueología y bioantropología de las Tierras Bajas, Universidad de la República, Faculdad de Humanidades y Ciencias de la Educación, Montevideo, p. 81-106.

Politis Gustavo et Mariano BonOMO

2012, « La entidad arqueológica Gota-Malabrigo (Ríos Paraná y Uruguay) y su filiación Arawak », Revista de arqueología, 25, p. 10-46.

Prieto Oscar, Alvaro Alvarez, Gerardo Arbenoiz, Juan De los Santos

1970, Informe preliminar sobre investigaciones arqueológicas en [sic] el Departamento de Treinta y Tres, R.O. Uruguay, Instituto Anchietano de Pesquisa (Publicacoes avulsas do Instituto anchietano de pesquisas, 18), Sao Leopoldo.

Schmitz Ignacio

1976, Sitios de Pesca Lacustre en Río Grande, Universidade Pontifica de Río Grande do Sul, Porto Alegre. 
Schmitz Ignacio et Jorge BAEZA

1980, « Santa Victoria do Palmar. Una tentativa de evolución ambiental, el ambiente del Arroyo Chuy y sus vinculaciones con la problemática de los cerritos », in Actas del VII Congreso nacional de Arqueología Uruguaya, Centro de Estudios Arqueológicos, Montevideo, p. 11-33.

Serrano Antonio

1972, Líneas fundamentales de la arqueología del litoral, Instituto de Antropología, Córdoba.

SuÁREZ Rafael

2010, Arqueología durante la transición Plesitoceno-Holoceno: componentes paleoindios, organización de la tecnología lítica y movilidad de los primeros americanos en Uruguay, thèse de doctorat, Universidad de la Plata, La Plata.

SuÁrez Rafael et José LóPez MAzz

2003, « Archaeology of the Pleistocene-Holocene transition in Uruguay: an overview », Quaternary international, 109-110, p. 65-76.

STEWARD Julian (éd.)

1946, Handbook of South American Indians, vol. 3. The tropical forest tribes, Smithsonian Institution (Bulletin of the Bureau of American Ethnology, 143), Washington.

TADDEI Antonio

1987, « Algunos aspectos de la arqueología prehistórica del Uruguay », in Lautaro Núñez A. et Betty Jane Meggers (éd.), Investigaciones paleoindias al sur de la línea ecuatorial, Instituto de Investigaciones Arqueológicas, Universidad del Norte (Estudios atacameños, 8), San Pedro de Atacama, p. 2-93. 
